\title{
Vibration Signal Analysis of Chimney Blasting Based on HHT
}

\author{
Sen HUANG ${ }^{\text {a,b,1 }}$, Linna LI ${ }^{\mathrm{a}, \mathrm{b}}$, Dongwang ZHONG ${ }^{\mathrm{a}, \mathrm{b}}$, Li HE $^{\mathrm{a}, \mathrm{b}}$ and Jianfeng SI ${ }^{\mathrm{a}, \mathrm{b}}$ \\ ${ }^{a}$ College of Science, Wuhan University of Science and Technology, \\ Wuhan 430065,Hubei, China \\ ${ }^{\mathrm{b}}$ Hubei Province Key Laboratory of Systems Science in Metallurgical Process, \\ Wuhan 430065, Hubei, China
}

\begin{abstract}
In the blasting demolition processs of high-rise structures, the impact of blasting vibration to the environment and objects to be protected must be effectively controlled, so the blasting vibration signal is deeply analyzed [1]. In this paper, the blasting vibration signal of a chimney is analyzedbased on HHT. The blasting vibration signal is denoised by Empirical Mode Decomposition (EMD)-wavelet threshold, then using Hilbert-Huang Transform (HHT) [2] the measured blasting vibration waveform Hilbert spectrum, marginal spectrum and instantaneous energy graph are draw to analyze the chimney blasting vibration. The results show that the denoising effect of EMD-wavelet threshold is good for blasting vibration signal [3]. HHT method has a good feature identification ability when processing vibration signals, and can reflect the characteristics of data more comprehensively and accurately, which provides convenience for the study of vibration signal data.
\end{abstract}

Keywords. Vibration measurement distance, HHT, EMD-Wavelet threshold denoising, the instantaneous signal

\section{Introduction}

With the development and progress of the society, people pay more and more attention to the impact of the development of industry on human living environment with the development of high technology. With the development of industry, waste gas, noise, sewage and other harmful substances will also follow. While enjoying the convenience brought by industrial development, human beings are also suffering from the harm brought by industry. In many factories, the chimney has begun to be dismantled, most of the chimney is carried out in the form of blasting due to the architectural characteristics of the chimney itself.And the chimney is often built in the factory, there will be more equipment, workshop next to the blasting environment is more complex, blasting range, chimney collapse direction has more stringent requirements. In the process of blasting the chimney, the blasting energy will not only affect the work of the chimney, but also cause a part of the blasting energy to the external environment [4]. There are rich data information in the signal of blasting vibration which is worthy of analysis, so it is very necessary to process the signal of blasting vibration. In traditional

\footnotetext{
${ }^{1}$ Linna Li, College of Science, Wuhan University of Science and Technology, Wuhan 430065,Hubei, China; E-mail: 12276086@qq.com.
} 
signal processing, Fourier Transform (FFT) is often used [5]. However, in Fourier Transform, the time domain of the collected signal is treated as a whole, and the signal is transformed into the frequency domain. The change of the signal is explained by its frequency. The disadvantage is that it cannot reflect the local signal information. If the signal is unstable or nonlinear, Fourier transform has certain limitations in the analysis of signal problems [6]. After The Fourier transform, in view of the limitations of The Local analysis of Fourier, the theory of wavelet analysis emerged, until today in the processing of signal analysis, wavelet analysis is still a commonly used means of signal analysis. However, wavelet analysis also has drawbacks. Its essential principle can be regarded as adding time window on the basis of Fourier transform, which is a Windowadjustable Fourier transform. At the same time, it faces the problem of how to select the wavelet basis. Hilbert-Huang Transform (HHT) was proposed by Norden E. Huang et al in 1998. HHT consists of two parts [7]. The first part is Empirical Mode Decompo-sition (EMD) and the second part is Hilbert Spectrum Analysis (HSA).

\section{Introduction to HHT Principle}

According to the characteristics of the data, HHT will carry out empirical mode decomposition (EMD) of the data, decompose and obtain several IMF components. Hilbert transformation is carried out on them to obtain Hilbert THREEDIMENSIONAL diagram, instantaneous energy spectrum, etc., which can intuitively reflect the data information and characteristics in the instantaneous state and nonstationary state [8].

\subsection{Empirical Mode Decomposition (EMD)}

The intrinsic mode function (IMF for short) obtained after EMD decomposition must first meet the following two conditions [9]:

(1) In the whole data series, the number of extremal points is equal to or at most 1 apart from the number of zero crossings.

(2) At any point on the signal, the mean values of the envelope determined by the local maximum point and the envelope determined by the local minimum point are both 0 , that is, the signal is locally symmetric on the time axis.

This method also has two purposes:

(1) Remove Superimposed Waves

(2) Make the waveform more symmetrical

The EMD implementation steps are as follows:

Firstly, the maximum value and minimum value of the signal $x_{1}(t)$ to be analyzed are found, and then the cubic spline function is used to interpolate it and fit the upper and lower envelope of the signal $x_{1}(t)$ to be analyzed. After calculation, the mean value of the upper and lower envelope is $m_{1}(t)$ Subtracting the mean value $x_{1}(t)$ from $m_{1}(t)$ gives the function $h_{1}(x)$ :

$$
h_{1}(t)=x_{1}(t)-m_{1}(t)
$$


Due to the different characteristics of the signals, $h_{1}(x)$ may not conform to the definition of IMF component. If not, $h_{1}(x)$ shall be put into the formula for repeated calculation:

$$
h_{i}(t)=h_{i-1}(t)-m_{i-1}(t)
$$

Repeat $i$ times until $h_{i}(t)$ meets the IMF definition. When the definition conditions of IMF are met, it is denoted as $c_{1}(t)$, and the remaining signal $x_{1}(t)$ is obtained by subtractsfr $h_{1 i}(t)$ om $r_{1}(t)$ :

$$
r_{1}(t)=x_{1}(t)-\mathrm{c}_{1}(t)
$$

Similarly, $r_{1}(t)$ is taken as A group of new data to be analyzed and brought into the process of modal decomposition. After repeated operations, the following results can be obtained:

$$
r_{i}(t)=r_{i-1}(t)-\mathrm{c}_{i}(t)
$$

when $r_{1}(t)$ is A monotone function, that is, when it is no longer possible to decompose IMF components from the data, the modal decomposition ends.

$$
r_{i}(t)=r_{i-1}(t)-\mathrm{c}_{i}(t)
$$

At this point, the original signal $x_{1}(t)$ to be analyzed can be obtained:

$$
x_{1}(t)=\sum_{i=1}^{n} \mathrm{c}_{i}(t)+r_{n}(t)
$$

where, $\mathrm{n}$ is the number of decomposed IMF components, and $r_{n}(t)$ is the residual, representing the average trend of the original signal $x_{1}(t)$.

\subsection{The Hilbert Transform}

After EMD decomposition, several IMF components are obtained, the instantaneous frequency of each IMF component can be obtained by Hilbert transformation, and the Hilbert spectrum of the whole set of data can be obtained by integrating the instantaneous frequency of all IMF components. 


\section{Distribution of Buildings Around the Chimney and Vibration Measurement Points}

To the east of the chimney is a factory road and the separation wall between the factory and the outside world. The wall is $3 \mathrm{~m}$ high, and the chimney is $16 \mathrm{~m}$ away from the wall.Outside the wall is a simple road to the river and a sewage treatment plant across the road. The south of the chimney is mainly a part of the bulk material workshop of the pelletizing plant; The western part of the chimney is the bulk material workshop of the pelletizing plant, about $15 \mathrm{~m}$ away from the chimney; The ring cooler is about $76 \mathrm{~m}$ away from the chimney. There is a clearing due north of the chimney, $43 \mathrm{~m}$ wide; $124 \mathrm{~m}$ due north of the chimney, there is an abandoned iron house, next to the iron house is the fine bin; On the west side of the vacant lot is the abandoned phase ii fine silo, which is a reinforced concrete frame workshop, $21.5 \mathrm{~m}$ away from the chimney due north axis; The east side is the wall of the factory, $21.5 \mathrm{~m}$ away from the axis due north of the chimney.

The type of vibration meter was Mini-BLAST i blasting vibration meter.Are measuring points 1 and 2 respectively, as shown in figure 1 .

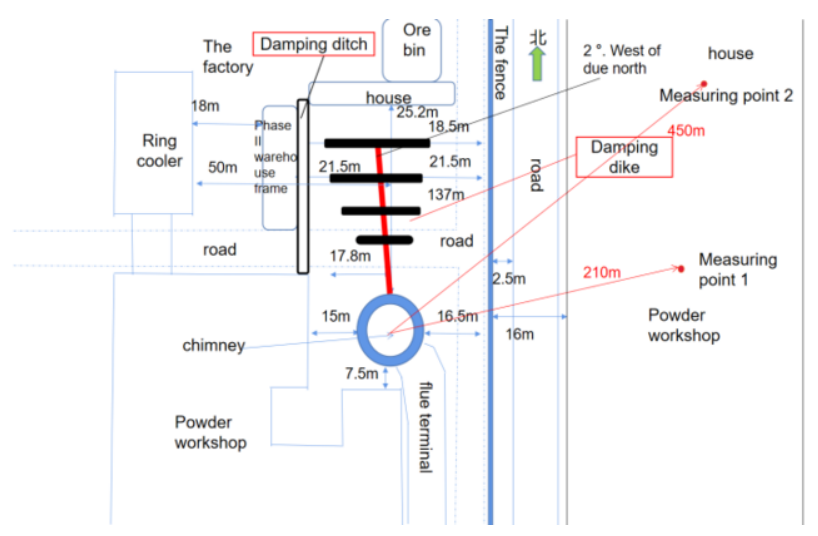

Figure 1. Distribution map of measuring points.

\subsection{Chimney Structure}

The pipe body of the chimney to be removed is a three-layer structure of reinforced concrete cylinder wall, insulation layer and inner lining (see figure 2).

The inner and outer sides of the concrete are reinforced.The height of the cylinder is less than or equal to $15 \mathrm{~m}$, the outer and inner vertical bars are $\varphi 18$, the outer and inner bars of the annular reinforcement are $\varphi 18$ and $\varphi 14$, the steel grid is $20 \times 20 \mathrm{~cm} 2$, and the concrete grade is $\mathrm{C} 30$. The bottom thickness of reinforced concrete cylinder wall is $40 \mathrm{~cm}$ (cylinder height $\leq 15 \mathrm{~m}$ ), the wall thickness decreases with the increase of height, and the top wall thickness is $18 \mathrm{~cm}$.

The chimney is $120 \mathrm{~m}$ upward from the horizon, with an outer diameter of $12.3 \mathrm{~m}$ and an inner diameter of $11.2 \mathrm{~m}$ at the bottom of the surface; The top outer diameter is about $6.3 \mathrm{~m}$ and the inner diameter is $5.9 \mathrm{~m}$. The bottom is provided with a rectangular outer layer and an arched internal flue opening, which is connected with a rectangular steel flue (see figure 3). The outer height of the flue is $11.2 \mathrm{~m}$ and the inner height is $9.7 \mathrm{~m}$. 


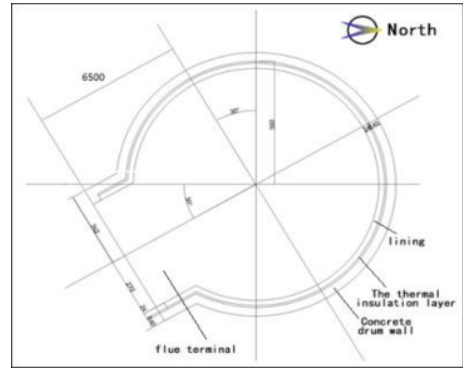

Figure 2. Cross-section of bottom of chimney.

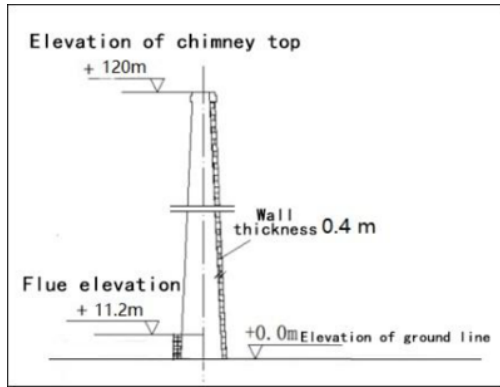

Figure 3. Chimney profile.

\section{Analysis of Blasting Vibration Signal}

In this blasting chimney project, vibration measuring points were set up in sewage treatment plant and civil houses respectively. Each vibration measuring instrument measured data from three directions: radial (x), tangential $(\mathrm{Y})$, vertical $(\mathrm{Z})$. The results after FFT preliminary transformation are shown in the table.It can be seen from table 1 that FFT dominant frequency and vibration velocity peak value in the $\mathrm{z}$ direction of measuring point 2 are relatively larger than those of $X$ and $Z$. Therefore, data in the vertical direction of measuring point 2 are selected for processing and analysis in this paper.

Table1. Information table of measurement points

\begin{tabular}{lllllll}
\hline \multirow{2}{*}{$\begin{array}{l}\text { Measuring } \\
\text { point }\end{array}$} & \multicolumn{3}{l}{ FFT frequency/Hz } & \multicolumn{4}{l}{ Peak vibration velocity/(mm· $\left.{ }^{-1}\right)$} \\
\cline { 2 - 7 } & $\mathrm{X}$ & $\mathrm{Y}$ & $\mathrm{Z}$ & $\mathrm{X}$ & $\mathrm{Y}$ & $\mathrm{Z}$ \\
\hline 1 & 62.5 & 93.2 & 100.6 & 1.7037 & 2.3081 & 4.5472 \\
2 & 63.9 & 60.2 & 18.7 & 0.1831 & 0.4388 & 0.9083 \\
\hline
\end{tabular}

\subsection{Signal Denoising}

For the blasting vibration signal, no signal can be completely independent of the surrounding environment, and there will be more or less the influence of the surrounding environment on its signal. In order to accurately analyze the vibration signal, the original signal denoising processing is essential.

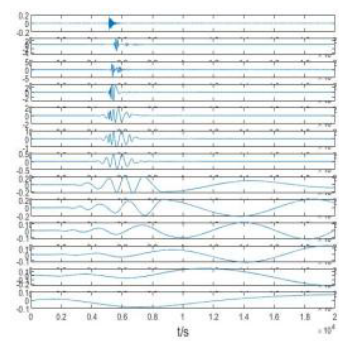

Figure 4. The EMD decomposition. 
First, the original signal was decomposed by EMD. As shown in figure 4, 13 IMF components were obtained after decomposition, and the frequencies of these 13 components decreased layer by layer. In the 13th component there is no longer a full period. It can be seen that EMD decomposition can be performed according to the characteristics of the data itself, and the decomposition will automatically stop when the whole cycle is not satisfied.Among the 13 IMF components, the frequency of the first group component is too high and the amplitude is low, which does not conform to the characteristics of vibration signals, and is a noise signal. Group 2 and Group 3 have higher frequencies and amplitudes. In the traditional denoising method, the first component is directly abandoned and the remaining components are reconstructed.In order to better remove the noise signal, this paper adds wavelet denoising method to the traditional denoising method.Wavelet denoising method: The signal is decomposed with the wavelet basis (DB4), and the decomposition layer is 5. The decomposed highfrequency components are denoised by threshold value, and the low frequency components are retained. Then the signal is restructured by denoising, and the denoising can be completed.Threshold: Can be obtained using the MATLAB function ddencmp.

In this paper, db4 wavelet basis function is used to decompose the IMF components of the second group, the third group and the fourth group. The number of decomposition layers is 5 . After de-noising, they are reconstructed with the remaining 9 IFM components to obtain complete de-noised vibration signals. Figure 5 shows the schematic diagram of the noise signals separated by the traditional method and the addition of wavelet threshold de-noising. From figure 6, we can see that the addition of wavelet threshold denoising does not have any influence on the peak value of vibration signal in the original signal. From figure 7 , we can see that the peak of the vibration signal that removes the noise has no effect. When high-frequency and low-amplitude signals are removed, blasting vibration signals are effectively retained, avoiding the problem of signal distortion caused by denoising.

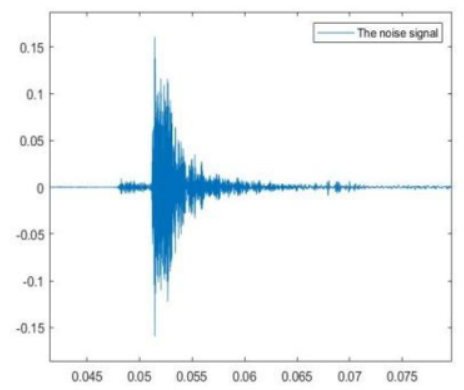

Figure 5. The noise signal.

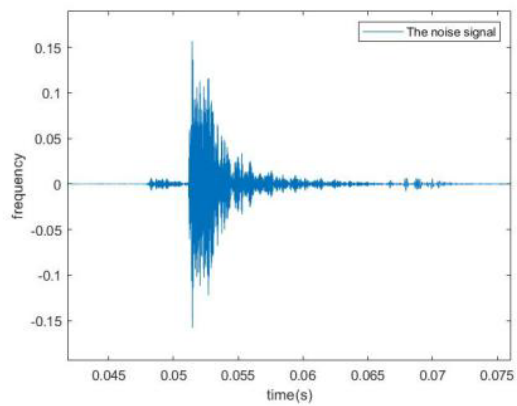

Figure 6. The noise signal. 

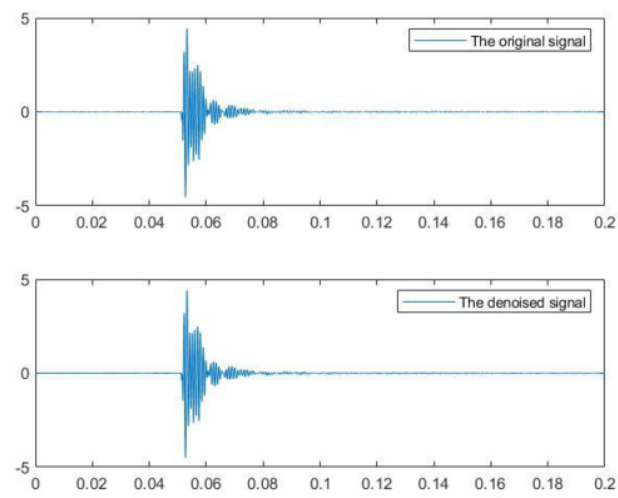

Figure 7. Contrast diagram of original signal and de-noised signal.

\subsection{Three-dimensional Hilbert spectrum Analysis}

After the noise in the signal is removed, Hilbert transformation is performed on the denoised signal to obtain the THREE-DIMENSIONAL Hilbert spectrum (figure 8). It can be intuitively seen from the figure that the vibration frequency of blasting is within, and the frequency of the maximum amplitude is $62.5 \mathrm{~Hz}$.

When the time is $0.05 \mathrm{~s}$, the maximum amplitude appears directly, and gradually weakens when the time is close to $0.1 \mathrm{~s}$. Compared with Fourier transform analysis, three-dimensional Hilbert spectrum analysis can reflect the relationship between time, frequency and amplitude simultaneously.

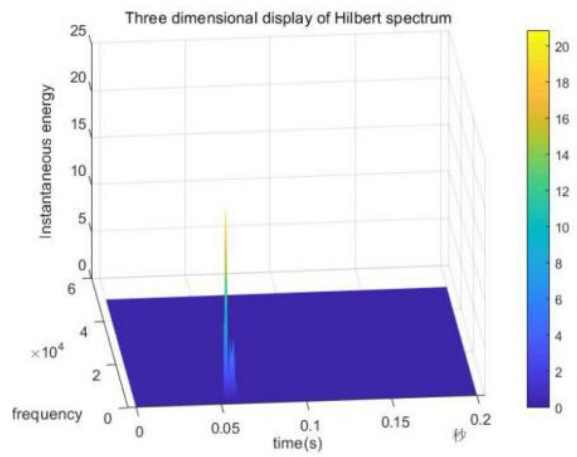

Figure 8. THREE-DIMENSIONAL Hilbert spectrum.

\subsection{The Marginal Spectrum}

In order to observe the instantaneous energy generated by blasting vibration more intuitively, the instantaneous energy spectrum is drawn by using HHT transform. As shown in figure 9, it can be seen that the maximum energy generated by blasting is $0.051 \mathrm{~s} \sim 0.057 \mathrm{~s}$, and the energy generated by blasting is gradually weakened in $0.057 \mathrm{~s} \sim 0.061 \mathrm{~s}$. The larger the amplitude, the faster the vibration speed, the two are proportional. When blasting $0.051 \mathrm{~s} \sim 0.057 \mathrm{~s}$ to the most destructive power of the 
chimney, at the same time, the impact on the surrounding buildings is also the biggest. According to the comparison between the instantaneous energy spectrum and the vibration signal diagram after denoising, it can be clearly seen that the peak values of the two are in one-to-one correspondence, indicating that THE HHT method has good signal identification ability for processing unstable signals.
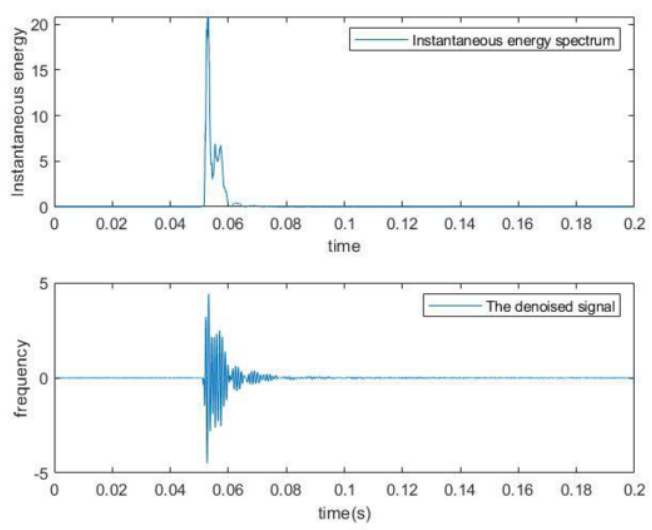

Figure 9. Instantaneous energy spectrum.

\section{Conclusion}

(1) For blasting vibration signals, in view of the complexity of the blasting environment, it is very necessary to conduct noise removal before signal analysis. After THE DECOMPOSITION of EMD, the traditional denoising and wavelet threshold denoising are combined to keep the required vibration signal while denoising, effectively avoid the denoising distortion, and the vibration signal processing has a good effect.

(2) In the blasting process, the vibration signal has no lasting fluctuation.At the moment of blasting, the energy reaches the maximum value and weakens to a slight fluctuation in a short time. The amplitude is mainly distributed at $62.5 \mathrm{~Hz}$, from $62.5 \mathrm{~Hz}$ to $687.5 \mathrm{~Hz}$ to the end, the amplitude decreases significantly, and tends to 0 after $1500 \mathrm{HZ}$. It is concluded that the energy distribution is concentrated in the low frequency part, which accords with the distribution law of blasting vibration.

(3) The instantaneous energy spectrum is used to identify the initiation time of the chimney, and the comparison with the theoretical value shows that the results are generally consistent, which verifies the feasibility of the method to identify the chimney blasting, and indicates that the HHT method has good signal resolution and recognition ability.

(4) HTT can accurately and multifaceted reflect the characteristics of data when processing unsteady nonlinear signals, which provides convenience for data research. Vibration signals generated in the blasting process are often unstable. Therefore, compared with Fourier transform and wavelet analysis, HHT is a better method for processing blasting vibration signal data at present. 


\section{References}

[1] Ji S, Xie W P, Wang L. Experimental study on characteristics and propagation law of blasting vibration and collapse vibration.Vibration and Shock. 2018 Nov, 37(11): 195-201.

[2] Zhong MS, Long Y, Xie MJ. Comparative analysis of collapse vibration and blasting vibration of demolition blasting in longhai building. Engineering Blasting. 2009 Dec; 15(4): 58-61.

[3] Dai J, Wang GZ, et al. Demolition of $45 \mathrm{~m}$ brick chimney in complex Environment by Blasting. Engineering Blasting. 2015 Apr; 21(2): 36-39.

[4] Du K, Fang X. Generalized dimension based on morphological covering for blasting vibration signal processing. 2017 Jun: 1755-1315.

[5] Wu XG. Demolition and blasting of a waste brick and concrete chimney in complex environment. China Mining Industry. 2014 Sep; 23(9): 149-151.

[6] Xie QM, Long Y, Zhong MS, et al. Application of SGWT in signal-noise separation of blasting vibration signal. Journal of Vibration And Shock. 2012 Sep; 31(1): 24-28.

[7] Liu XL, Yuan HP, Zheng X, et al. Time-frequency energy analysis of blasting vibration signal based on HHT. Journal of Hefei University of Technology (Natural Science), 2019 Jun; 42(06): 779-784.

[8] Wang L, Zhang L. Demolition blasting of $60 \mathrm{~m}$ high cooling tower in complex environment. Blasting. 2011 Jun; 28 (2):76-78.

[9] Yang ZB, Teng C. Research status and existing problems of building demolition blasting. Management \& Technology. 2016 Oct; (10) : 54-55. 